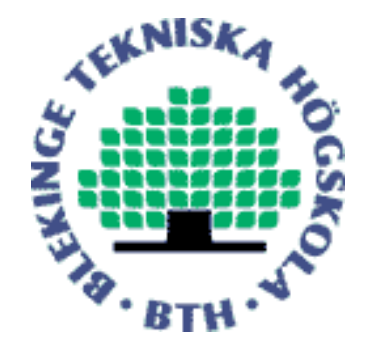

Copyright ( 2008 IEEE. Citation for the published paper:

Rakus-Andersson Elisabeth, Salomonsson Maria, Zettervall Hang "Ranking of Weighted Strategies in the Two-player Games with Fuzzy Entries of the Payoff Matrix."

Hybrid Intelligent Systems 2008 - HIS 2008, 2008, Barcelona, Spain

This material is posted here with permission of the IEEE. Such permission of the IEEE does not in any way imply IEEE endorsement of any of BTH's products or services Internal or personal use of this material is permitted. However, permission to reprint/republish this material for advertising or promotional purposes or for creating new collective works for resale or redistribution must be obtained from the IEEE by sending a blank email message to pubspermissions@ieee.org.

By choosing to view this document, you agree to all provisions of the copyright laws protecting it. 


\section{Ranking of Weighted Strategies in the Two-Player Games with Fuzzy Entries of the Payoff Matrix}

\author{
Elisabeth Rakus-Andersson ${ }^{\dagger}$ \\ Blekinge Institute of \\ Technology, Department of \\ Mathematics and Science \\ S-37179 Karlskrona, Sweden \\ Elisabeth.Andersson@bth.se
}

\author{
Maria Salomonsson \\ Blekinge Institute of \\ Technology, Department of \\ Signal Processing \\ S-37225 Ronneby, Sweden \\ Maria.Salomonsson@bth.se
}

\author{
Hang Zettervall \\ Blekinge Institute of \\ Technology, Department of \\ Mathematics and Science \\ S-37179 Karlskrona, Sweden \\ Hang.Zettervall@bth.se
}

\begin{abstract}
We expand the classical model of a two-player game to select the best strategies, whose action is expected to maintain the values of a certain variable on the neutral level. By inserting fuzzy sets as payoff values in the game matrix we facilitate the procedure of formulations of payoff expectations by players. Instead of making difficult decisions about the choice of accurate numerical entries of the matrix the players are able to use words, which should simplify a communication between them when designing the preliminaries of the game. The players also have the possibility of making a ranking of their favorite strategies.
\end{abstract}

\section{Introduction}

Nowadays, a large number of contributions to fuzzy games can be found in literature, e.g., in $[3,5,6,7,8$, 10]. The majority of models are based on the classical crisp method of maintaining the equilibrium [1, 2], even though assumptions of fuzzy approaches differ considerably from each other. We can mention the models based on coalitional structures of a game [3], the models involving multi-criteria of fuzzy decision making [5], the algorithms using fuzzy rules of inference [7], the algorithms applying axiomatization of the game core by means of consistency [8], or noncooperative games with fuzzy parameters [10].

We sketch an original simple counterpart of the two-player game with imprecise coefficients of the payoff matrix [11] and weighted strategies. The execution of the algorithm provides us with a sample of ranked strategies selected from all tactics prepared by the players, on condition that an objective of the game is to keep the balance status of a profit variable.

In Section 2 we discuss a theoretical aspect of the proposed method to add the indices of strategy importance in Section 3. We practise the action of our proposal action on medical and technical examples in Sections 4 and 5.

\section{The game with imprecise payoff values}

Assume the existence of two players $A$ and $B$ ( $A$ and $B$ may represent teams) who adopt their action plans called strategies to win a zero-sum-game. The row player $A$ uses his strategies $R_{r}, r=1, \ldots, m$, whereas the column player $B$ makes a selection among his strategies $K_{k}, k=1, \ldots, n$. Suppose further that $A$ and $B$ choose the strategies independently of each other. Both $A$ and $B$ select their strategies $R_{r}$ and $K_{k}$ respectively, which results in a gain being obtained by one of them and a loss by the other. The gains and the losses are preliminarily determined for each pair of strategies $\left(R_{r}\right.$, $K_{k}$ ) due to a profit plan yielded by a payoff matrix $P=$ $\left\{\left(\left(R_{r}, K_{k}\right), \quad \mu_{P}\left(R_{r}, K_{k}\right)\right)\right\}$, where all $\mu_{P}\left(R_{r}, K_{k}\right)$ represent fuzzy sets attached to verbal descriptions of the profit, $r=1, \ldots, m, k=1, \ldots, n$.

After reminding the reader of the basic assumptions of the classical game model let us explain our interpretations of profits, gains and losses.

By "profit" we mean the deviations of values of a certain variable in either positive or negative directions. We wish to preserve the achieved variable values on a balance level near zero after using the most accurate strategies for that purpose. $A$ thus aims at finding the strategy that, used against $B$ 's strategies,

\footnotetext{
${ }^{\dagger}$ This work is partially granted by the Swedish Royal Academy of Sciences
} 
allows $A$ to keep his gain near the zero balance level. $B$ intends to test his strategies contra $A$ 's providing him with a loss near zero.

The players wish to determine the matrix $P$ as a collection of words at the initial stage of the game. Each entry of $P$ constitutes a prognosis of the change in the variable status when letting the pair $\left(R_{r}, K_{k}\right)$ affect the variable values. Let us differentiate changes in the profit variable as terms of a linguistic variable "changes of the profit variable" $=\{$ " $n b$ " = "negative big”, " $n$ ” = "negative”, "ns” = "negative small”, " $z$ " = "zero", "ps” = "positive small”, " $p$ ” = "positive”, “pb” = "positive big" $\}$. Each notion is assimilated with a fuzzy set that constitutes the primary entry of $P$ intended for the pair $\left(R_{r}, K_{k}\right)$ to be finally translated in $\mu_{P}\left(R_{r}, K_{k}\right) \in[0,1]$.

All $\mu_{P}\left(R_{r}, K_{k}\right)$, observed in $P$, denote the gains of $A$, provided that the gains are related to $A$ 's ability to increase the variable values. Thus, larger $\mu_{P}\left(R_{r}, K_{k}\right)$ correspond to positively directed changes of the profit variable for $A$, whereas lower $\mu_{P}\left(R_{r}, K_{k}\right)$ in the $A$ 's case are associated with negative deviations in the variable range. The same quantities of $\mu_{P}\left(R_{r}, K_{k}\right)$ are interpreted for $B$ as his capacity to affect the variable values negatively, which answers to his losses. The presence of $\mu_{P}\left(R_{r}, K_{k}\right)=0.5$ in the matrix emphasizes the existence of the zero level in the variable value estimation when using $R_{r}$ against $K_{k}$. If, e.g., $\mu_{P}\left(R_{r}, K_{k}\right)=0.25$ then $B$ 's power of producing negative effects in the variable range is stronger than A's possibility to balance the range positively.

All fuzzy sets are impacted over the interval of variable changes $\left[a_{\min }, a_{\max }\right]$. Suppose $a_{\min }<0, a_{\max }>$ 0 . We define three atomic expressions in $\left[a_{\min }, a_{\max }\right]$, i.e., "the leftmost" = "negative", "in the middle" = "zero" and "the rightmost" = "positive" to add the hedges "big" and "small" to "negative" and "positive". The constrains of the "negative" family of sets preceded by "big" and "small" are the s-class functions $1-s\left(x, \frac{2}{3} a_{\min } \delta, \frac{1}{2} a_{\min } \delta, \frac{1}{3} a_{\min } \delta\right)$ defined as [9]

$y=\mu_{\text {"hedge negative" }}(x)=$
$\begin{cases}1 & \text { for } x \leq \frac{2}{3} a_{\min } \delta, \\ 1-2\left(\frac{x-\frac{2}{3} a_{\min } \delta}{\left(\frac{1}{3} a_{\min }-\frac{2}{3} a_{\min }\right)}\right)^{2} & \text { for } \frac{2}{3} a_{\min } \delta<x \leq \frac{1}{2} a_{\min } \delta, \quad(1 \\ 2\left(\frac{x-\frac{1}{3} a_{\min } \delta}{\left(\frac{1}{3} a_{\min }-\frac{2}{3} a_{\min }\right) \delta}\right)^{2} & \text { for } \frac{1}{2} a_{\min } \delta<x<\frac{1}{3} a_{\min } \delta, \\ 0 & \text { for } x \geq \frac{1}{3} a_{\min } \delta .\end{cases}$

The application of the parameter $\delta$ has either the narrowing ( $\delta>1$ ) or the widening ( $\delta<1$ ) effects for the supports of fuzzy sets generated by "negative" if $\left[a_{\min }, 0\right]$ contains negative quantities. The value of $\delta=$ 1 has no influence on the support of "negative". In order to implement the membership functions of the left group of expressions belonging to "changes of the profit variable" we suggest $\delta=1.5$ for "negative big”, $\delta=1$ for "negative” and $\delta=0.5$ for "negative small”.

For the "positive" collection of sets we derive the membership functions $s\left(x, \frac{1}{3} a_{\max } \delta, \frac{1}{2} a_{\max } \delta, \frac{2}{3} a_{\max } \delta\right)$ being

$$
\begin{aligned}
& y=\mu_{\text {"hedge positive" }}(x)= \\
& \begin{cases}0 & \text { for } x \leq \frac{1}{3} a_{\max } \delta, \\
2\left(\frac{x-\frac{1}{3} a_{\max } \delta}{\left(\frac{2}{3} a_{\max }-\frac{1}{3} a_{\max }\right) \delta}\right)^{2} & \text { for } \frac{1}{3} a_{\max } \delta<x \leq \frac{a_{\max }}{2} \delta, \\
1-2\left(\frac{x-\frac{2}{3} a_{\max } \delta}{\left(\frac{2}{3} a_{\max }-\frac{1}{3} a_{\max }\right) \delta}\right)^{2} & \text { for } \frac{a_{\max }}{2} \delta<x<\frac{2}{3} a_{\max } \delta, \\
1 & \text { for } x \geq \frac{2}{3} a_{\max } \delta,\end{cases}
\end{aligned}
$$

with $\delta=0.5$ assigned to "positive small", $\delta=1$ corresponding to "positive" and $\delta=1.5$ creating “positive big”. Even for $\left[0, a_{\max }\right]$, containing positive values, we observe the result of a shrunk support for $\delta>1$ and the effect of a stretched support if $\delta<1$ when comparing to the support of "positive".

The impact laid on "zero" consists of two s-class functions joined together by an expression

$$
\begin{aligned}
& y=\mu_{\text {"zero" }}(x)= \\
& \begin{cases}s\left(x, \frac{1}{3} a_{\min }, \frac{1}{6} a_{\min }, 0\right) & \text { for } x \leq 0, \\
1-s\left(x, 0, \frac{1}{6} a_{\max }, \frac{1}{3} a_{\max }\right) & \text { for } x \geq 0 .\end{cases}
\end{aligned}
$$

In Fig. 1 we plot membership functions of the fuzzy sets from "changes of the profit variable" for, e.g., $\left[a_{\min }, a_{\max }\right]=[-9,9]$.

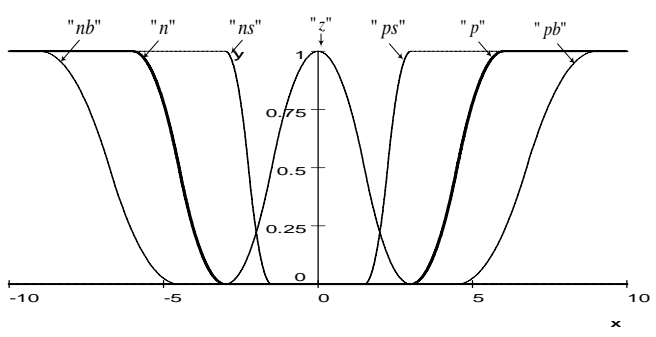

Figure 1. Fuzzy sets representing the terms of "changes of the profit variable" in [-9, 9] 
In order to design the payoff matrix in the numerical shape, we extract one value from each set support as an indicator of the set. We accept element $x$ of the set support that is assimilated to a certain border of the set's membership function. We can establish the boundary value $x$, as the $x$-coordinate of an intersection point between the line $\mu_{\text {set }}(x)=1$ and a part of the membership function in which $\mu_{\text {set }}(x)<1$.

The expressions coming from "negative" have thus the defuzzified values, pointing out the sets, determined as $x=\frac{2}{3} a_{\min } \delta$ whereas the descriptions created by "positive" form the group with representatives equal to $x=\frac{2}{3} a_{\max } \delta$.

For the terms of the list "changes of the profit variable" we have implemented a procedure furnishing us with members of all descriptions stated as $x_{n n} n b^{\prime \prime}=a_{\min }, \quad x_{n n^{n}}=\frac{2}{3} a_{\min }, \quad x_{n n s "}=\frac{1}{3} a_{\min }, \quad x_{{ }^{\prime \prime}}=0$, $x_{n} p s^{n}=\frac{1}{3} a_{\max }, x_{n "} p^{\prime \prime}=\frac{2}{3} a_{\max }$ and $x_{n} p b^{n}=a_{\max }$.

We construct another fuzzy set "change" over $\left[a_{\min }\right.$, $\left.a_{\max }\right]$ stressed by a linear membership function

$$
\begin{aligned}
& \mu_{\text {"change" }}(x)= \\
& \left\{\begin{array}{lll}
0 & \text { for } & x<a_{\min }, \\
\frac{1}{2}\left(-\frac{x}{a_{\min }}+1\right) & \text { for } & a_{\min } \leq x \leq 0, \\
\frac{1}{2}\left(\frac{x}{a_{\max }}+1\right) & \text { for } & 0 \leq x \leq a_{\max }, \\
1 & \text { for } & x>a_{\max },
\end{array}\right.
\end{aligned}
$$

to find membership values for all representatives of terms from the list "changes in the profit variable". We list the results in Table 1

TABLE I

THE REPRESENTATIVES OF FuZZY SETS From

\begin{tabular}{|c|c|c|}
\hline Fuzzy sets & $x$ & 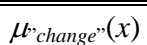 \\
\hline "negative big" & $x_{n n b "}=a_{\min }$ & 0 \\
\hline "negative" & $x_{n n}=\frac{2}{3} a_{\min }$ & 0.167 \\
\hline “negative small” & $x_{n n s "}=\frac{1}{3} a_{\min }$ & 0.333 \\
\hline “zero” & 0 & 0.5 \\
\hline “positive small” & $X_{" p s "}=\frac{1}{3} a_{\max }$ & 0.667 \\
\hline "positive" & $x_{n p^{\prime \prime}}=\frac{2}{3} a_{\max }$ & 0.833 \\
\hline “positive big” & $X_{" p b "}=a_{\max }$ & 1 \\
\hline
\end{tabular}
“CHANGES OF THE PROFIT VARIABLE"

In matrix $P$ the entries are predetermined by the

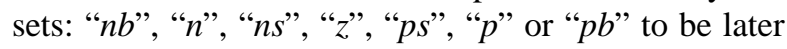
replaced by $\mu_{P}\left(R_{r}, K_{k}\right)$ being one of the values: 0 , $0.167,0.333,0.5,0.667,0.833$ or 1 . We can always extend the number of notions in the list in conformity with our needs.

Having obtained the numerical data in $P$ we try to rank $A$ 's and $B$ 's best strategies to let the players keep the variable quantities invariable near zero. $A$ thus maximizes his minimal influence on making the variable values positive when testing $R_{r}$ one by one. $B$ replies to $R_{r}$ with one strategy among $K_{1}, \ldots, K_{n}$, whose choice is unknown for $A$. The selection of the best strategy $R^{*}$ for $A$ is supported by a value $\mu_{A}\left(R^{*}\right)$ introduced by the formula

$$
\mu_{A}\left(R^{*}\right)=\max _{1 \leq r \leq m}\left(\min \left(\mu_{P}\left(R_{r}, K_{1}\right), \ldots, \mu_{P}\left(R_{r}, K_{n}\right)\right) .\right.
$$

If the value of $\mu_{A}\left(R^{*}\right)$ appears in row $r$ then strategy $R_{r}$, marking the row $r$, will be considered as the optimal strategy $R^{*}=R_{r}, r=1, \ldots, m$.

Since $B$ should minimize the largest decreases of values caused by his strategies $K_{k}$ then we seek the optimal $K^{*}$ strategy used against an unknown choice of $R_{1}, \ldots, R_{m}$ to maintain the profit around zero. We compute $\mu_{B}\left(K^{*}\right)$, which helps to recognize $K^{*}$, as

$\mu_{B}\left(K^{*}\right)=\min _{1 \leq k \leq n}\left(\max \left(\mu_{P}\left(R_{1}, K_{k}\right), \ldots, \mu_{P}\left(R_{m}, K_{k}\right)\right) . \quad\right.$ ) 6

Strategy $K^{*}=K_{k}$ will be accepted as $B$ 's optimal method used in the game if the value of $\mu_{B}\left(K^{*}\right)$ is found in column $k, k=1, \ldots, n$.

After having found $R^{*}$ and $K^{*}$ we determine the preliminary hierarchies of strategies for both players (there can be more than one strategy assigned to the player) remembering that the objective of the game is to prohibit the variable from either positive or negative extreme changes of its values.

\section{Unequal strategies supporting the game}

The players $A$ and $B$ can also make own judgments of importance concerning their favorite strategies used in the game. The preliminary ranking made in the end of Section 2 is expected to provide the players with useful hints to set preferences for their strategies, which will be followed by numerical values of weights assigned to strategies. For $A$ let us denote the weights by $\omega_{1}, \ldots, \omega_{m}$, and for $B$ the weights will be marked by $v_{1}, \ldots, v_{n}$. For A's $m$ strategies we adopt a procedure of obtaining an importance ratio scale due to [4]. Assume that for $m$ strategies we wish to construct a scale, rating them as to their importance for making the decision. We compare the strategies in pairs to judge the importance of the pair first component over the 
second one when affecting a neutral result of the game. If we confront strategy $R_{j}$ with strategy $R_{l}$, then we can assign the values $c_{j l}$ and $c_{l j}$ to the pair $\left(R_{j}, R_{l}\right)$ as follows, $j, l=1, \ldots, m$ :

(1) $c_{l j}=\frac{1}{c_{j l}}$,

(2) If $R_{j}$ is more important than $R_{l}$ then $c_{j l}$ gets assigned one of the numbers $1,3,5,7$ or 9 due to the difference of importance being equal, week, strong, demonstrated or absolute, respectively. If $R_{l}$ is more important than $R_{j}$, we will assign the value of $c_{l j}$.

Having obtained the above judgments an $m \times m$ matrix $C=\left(c_{j l}\right)_{j, l=1}^{m}$ is constructed.

A's weights $\omega_{1}, \ldots, \omega_{m}$ are decided as components of the eigen vector corresponding to the largest in magnitude eigen value of the matrix $C$. We normalize the weights $\omega_{r}, r=1, \ldots, m$, by dividing all of them by the largest weight $\omega_{\text {largest }}$. We suggest this simple operation to keep all $\omega_{r}$ within interval $[0,1]$. Let us denote the normalized $\omega_{r}$ by $\hat{\omega}_{r}=\frac{\omega_{r}}{\omega_{\text {largest }}}$. We assume that the higher value of $\hat{\omega}_{r}$ points out the more significant influence of $R_{r}$ on reaching the game objective. If we multiply entries of $P$, belonging to the row characterized by the strongest strategy, by the value of one then we will not change their magnitudes. Since we multiply other rows of $P$ by fractions belonging to $[0,1]$ then we will decrease the values placed in the rows. In these circumstances, after a selection of maximum of minimal values made by $A$, we still prefer the same strategy as before to be optimal. We thus suggest the introduction of weights $\hat{\omega}_{r}$ in matrix $P$ in the shape adapted to $A$, i.e., we design matrix $P_{A}=\left\{\left(\left(R_{r}, K_{k}\right),\left(\mu_{P}\left(R_{r}, K_{k}\right)\right) \cdot \hat{\omega}_{r}\right)\right\}, r=$ $1, \ldots, m, k=1, \ldots, n$. To select the optimal strategy $R^{*}$ of $A$ we estimate $\mu_{A}^{\text {eeighted }}\left(R^{*}\right)$ by modifying (5) as

$$
\begin{aligned}
& \mu_{A}^{\text {weighted }}\left(R^{*}\right)= \\
& \max _{1 \leq r \leq m}\left(\min \left(\mu_{P}\left(R_{r}, K_{1}\right) \cdot \hat{\omega}_{r}, \ldots, \mu_{P}\left(R_{r}, K_{n}\right) \cdot \hat{\omega}_{r}\right)\right.
\end{aligned}
$$

when assuming that $A$ does not know anything about $B$ 's importance priorities.

Another matrix $D=\left(d_{i, p}\right)_{i, p=1}^{n}$, determined in compliance with [4], sums up the effects of $B$ 's comparison made for importance degrees of $K_{1}, \ldots, K_{n}$. The expected weights $\hat{v}_{1}, \ldots, \hat{v}_{n}$ are obtained as normalized coordinates of the eigen vector associated with the largest real eigen value of $D$. $B$ still wants to find minimum of maximal values computed for entries of $P$ 's columns. In order to exhibit the values of column $k$ assisting $K^{*}$, as the most suitable for the choice of the minimal maximum number we multiply it by the least weight among $\hat{v}_{1}, \ldots, \hat{v}_{n}$. We reverse the order in the sequence of $\hat{v}_{1}, \ldots, \hat{v}_{n}$ as to their importance to assign the value of one to the weakest strategy and the least power to the strongest one. We denote strategy $\hat{v}_{k}$ by $\hat{v}_{k}^{\text {reversed }}$ after the replacement to implement $B$ 's version of the payoff matrix $P_{B}$ designed as $P_{B}=\left\{\left(\left(R_{r}, K_{k}\right),\left(\mu_{P}\left(R_{r}, K_{k}\right)\right) \cdot \hat{v}_{k}^{\text {reversed }}\right)\right\}, r$ $=1, \ldots, m, k=1, \ldots, n$. To select the optimal strategies $K^{*}$ that $B$ uses in his responses against $A^{\prime}$ s moves we evaluate $\mu_{B}^{\text {weighted }}\left(K^{*}\right)$ as another version of (6) proposed as

$$
\begin{aligned}
& \mu_{B}^{\text {weighted }}\left(K^{*}\right)=\min _{1 \leq k \leq n}\left(\operatorname { m a x } \left(\mu_{P}\left(R_{1}, K_{k}\right) \cdot \hat{v}_{k}^{\text {reversed }},\right.\right. \\
& \left.\ldots, \mu_{P}\left(R_{m}, K_{k}\right) \cdot \hat{v}_{k}^{\text {reversed }}\right) .
\end{aligned}
$$

We assume that $B$ is not familiar with $A$ 's expectations concerning strategies $R_{r}, r=1, \ldots, m$.

\section{Optimal strategies in the maintenance of blood pressure level}

A patient with coronary heart disease has to keep changes of systolic blood pressure near zero change level. The changes are expected to happen in $\left[a_{\min }, a_{\max }\right]=[-30 \mathrm{Hgmm}, 30 \mathrm{Hgmm}]$. Two groups of specialists $A$ and $B$ want to help him in maintaining the changes on the balance level, that is, near zero. $A$ prepares strategies $R_{1}, R_{2}, R_{3}$ (e.g., medicines, diets, physical exercises) contra $B$ 's responses $K_{1}, K_{2}, K_{3}$. Both $A$ and $B$ are aware of effects of their joined-inpair strategies. The effectiveness of the strategies is presented in the following matrix:

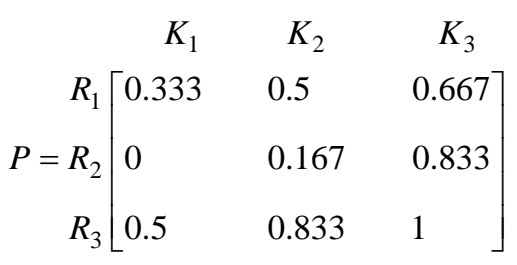

For $A$, due to (5), $\mu_{A}\left(R^{*}\right)=\max (\min (0.333,0.5$, $0.667), \min (0,0.167,0.833), \min (0.5,0.833,1))=0.5$. $A$ should thus prioritize the strategies as the sequence $R_{1} \approx R_{3} \succ R_{2}$ provided that $R_{i} \succ R_{j}$ means that $R_{i}$ acts 
better than $R_{j}$. We evaluate the optimal strategies for $B$ according to $\mu_{B}\left(K^{*}\right)=\min (\max (0.333,0,0.5)$, $\max (0.5,0.167,0.833), \max (0.667,0.833,1))=0,5$, emphasizing the order $K_{1} \approx K_{2} \succ K_{3}$.

The weights of strategies, being the factors of $A$ 's judgments in accordance with preferences $R_{1} \succ R_{3} \succ R_{2}$, are effects of priorities made for pairs of strategies in matrix $C$ stated as

$$
\begin{aligned}
& \begin{array}{lll}
R_{1} & R_{2} & R_{3}
\end{array} \\
& C=\begin{array}{r}
R_{1} \\
R_{2} \\
R_{3}
\end{array}\left[\begin{array}{lll}
1 & 7 & 5 \\
1 / 7 & 1 & 1 / 3 \\
1 / 5 & 3 & 1
\end{array}\right]
\end{aligned}
$$

The normalized coordinates of the eigen vector, associated with the largest eigen value, constitute the weights $\hat{\omega}_{1}=1, \hat{\omega}_{2}=0.111, \hat{\omega}_{3}=0.258$. We insert these values in the matrix $P$ to get its version as

$$
P_{A}=\left[\begin{array}{lll}
0.333 & 0.5 & 0.667 \\
0 & 0.019 & 0.092 \\
0.129 & 0.215 & 0.258
\end{array}\right]
$$

By (7), we obtain: $\mu_{A}^{\text {weighted }}\left(R^{*}\right)=\max [\min (0.333$, $0.5,0.667), \min (0,0.019,0.092), \min (0.129,0.215$, $0.258)]=\max [0.333,0,0.129]=0.333$, which even stronger confirms the advantage of $R_{1}$ in the process of keeping the blood pressure balance level.

Since $B$ sets his strategies in order $K_{2} \succ K_{1} \succ K_{3}$ then matrix $D$ takes a form of

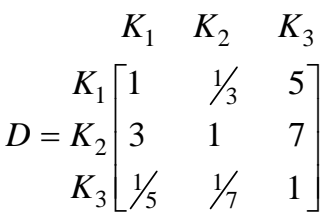

By means of $D$ we obtain $\hat{v}_{1}=0.43, \hat{v}_{2}=1$, $\hat{v}_{3}=0.11$. After reversing the order of weights according to rules described in Section 3 we determine $\hat{v}_{1}^{\text {reversed }}=0.43, \hat{v}_{2}^{\text {reversed }}=0.11, \hat{v}_{3}^{\text {reversed }}=1$, used to design matrix $P_{B}$ as

$$
P_{B}=\left[\begin{array}{lll}
0.143 & 0.056 & 0.667 \\
0 & 0.019 & 0.833 \\
0.215 & 0.093 & 1
\end{array}\right]
$$

In compliance with (8) $\mu_{B}^{\text {weighted }}\left(K^{*}\right)=\min$ $[\max (0.143,0,0.215), \max (0.056,0.019,0.093)$, $\max (0.667,0.833,1)]=\min [0.215,0.093,1]=0.093$. Since 0.093 belongs to column marked by $K_{2}$ then this strategy is recognized as optimal, which completely agrees with the prognosis previously made.

\section{The constant radio transmission rate in patient monitoring products modeled as a fuzzy game}

Wireless communication is increasingly being used in a variety of medical applications. Consider the following example of two proprietary wireless patient monitoring products using cognitive radio, whose main function is that of detecting and selecting appropriate frequency bands to be used for transmission.

If the aim is to keep the data transmission rate constant and equally divided amongst the monitoring products, the problem can be modeled as a two-player game, using a linguistically formulated payoff matrix. The patient monitor products are represented by players $A$ and $B$, choosing amongst different frequency bands, or channels (they may choose more than one channel) independently of each other. More data can be transmitted in the same period of time by using more channels. However, if several transmissions are attempted on the same channel, less data per second can be transmitted due to the overlap.

$A$ uses the strategies $R_{1}, R_{2}, R_{3}$, and $B$ the strategies $K_{1}, K_{2}, K_{3}$. All strategies refer to specific channel selection configurations which may be placed in order of precedence, thus weighted. The changes are expected to happen within $\left[\alpha_{\min }, \alpha_{\max }\right]=[-3 M b p s$, $3 M b p s]$. An illustrative payoff matrix can be formulated as:

$$
\begin{gathered}
K_{1} \\
P=R_{2} R_{2} \\
R_{3}
\end{gathered}\left[\begin{array}{lll}
0.5 & 0.5 & K_{3} \\
1 & 0.667 & 0.333 \\
0 & 0.333 & 0.667
\end{array}\right]
$$

From the payoff matrix we can evaluate $\mu_{A}\left(R^{*}\right)=$ 0.5 . A should hence use the preference $R_{1} \succ R_{3} \approx R_{2}$ to maximize his minimal profits, i.e. to keep a balanced 
data rate for both players. Evaluating $\mu_{B}\left(K^{*}\right)=0,667$, indicates that player $B$ should choose the strategies $K_{2}$ and $K_{3}\left(K_{2} \approx K_{3} \succ K_{1}\right)$ to give a transmission rate change to the advantage of player $A$.

If players $A$ and $B$ wish to rank their strategies in accordance with their preferences, we will introduce the judgment matrix $C$ for player $A$, and the judgment matrix $D$ for player $B$ designed as

$$
\begin{aligned}
& \begin{array}{llllll}
R_{1} & R_{2} & R_{3} & K_{1} & K_{2} & K_{3}
\end{array}
\end{aligned}
$$

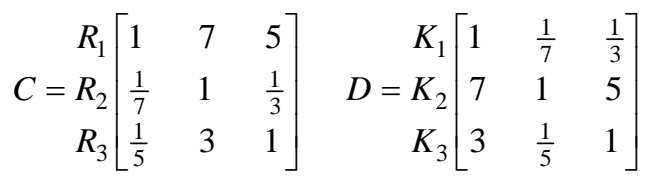

After the introduction of weights in $P$ we obtain:

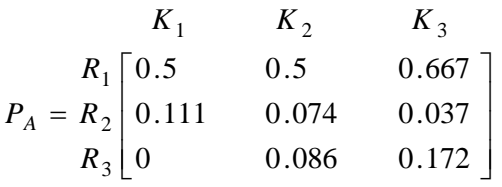

and

$K_{1}$
$P_{B}=R_{1}$
$R_{2}$
$R_{3}$$\left[\begin{array}{llc}0.5 & 0.055 & K_{3} \\ 1 & 0.073 & 0.143 \\ 0 & 0.037 & 0.287\end{array}\right]$

We may consequently evaluate the optimal strategies for players $A$ and $B$ as $\mu_{A}^{\text {weighted }}\left(R^{*}\right)=0.5$ and $\mu_{B}^{\text {weighted }}\left(K^{*}\right)=0.073$.

Thus player $A$ should use the strategy $R_{1}$ and player $B$ ought to prefer the strategy $K_{2}$ to keep the data transmission rate constant.

\section{Conclusions}

The attempt, made for the achievement of a balance level in the two-player game and supported by a fuzzy payoff matrix, results in the formulation of a method providing two players with the ranking of strategy effectiveness. The membership functions of fuzzy sets, assisting verbal expressions used in the description of variable change intensities, have been designed as parametric classes of s-functions. We hope that it should highlight the logical and elegant design of the mathematical scenario adopted in the discussion of the model proposed.
The strategies have also been furnished with weights confirming the players' beliefs in the effective powers of these tactics. This complement, added to the preferences, preliminarily made in the first part of the model, has unambiguously resulted in revealing the best strategies.

We suggest applications of the proposed version of two-player fuzzy games in the imprecise circumstances of variable changes, when a saddle point for strategies cannot be found.

\section{References}

[1] J. Nash, "Equilibrium Points in N-person Games”, Proc. Nat. Academy Sci., vol. 36, 1950, pp. 48-49.

[2] L. Shapley, "A Value for N-person Games”, Contributions to the Theory of Games. Princeton, NJ: Princeton Univ. Press, 1953, pp. 207-317.

[3] D. Butnariu, "Fuzzy Games: A Description of Time Concept”, Fuzzy Sets and Systems, vol. 1, 1978, pp. 181192.

[4] T. L. Saaty, "Exploring the Interface between Hierarchies, Multiplied Objectives and Fuzzy Sets”, Fuzzy Sets and Systems, vol. 1, 1978, pp. 57-68.

[5] Quian Song, and A. Kandel, "A Fuzzy Approach to Strategic Games”, IEEE Transactions on Fuzzy Systems, vol. 7, 1999, pp. 634-642.

[6] Kim Won Kyu, and Lee Kyoung Hee, "Generalized Fuzzy Games and Fuzzy Equilibria”, Fuzzy Sets and Systems, vol. 122, 2001, pp. 293-301.

[7] B. Arfi, "Linguistic Fuzzy-Logic Game Theory", Journal of Conflict Resolution, vol. 50, 2006, pp. 28-57.

[8] Yan-An Hwang, "Fuzzy Games: A Characterization of the Core", Fuzzy Sets and Systems, vol. 158, 2007, pp. 24802493.

[9] E. Rakus-Andersson, "The Parametric Modification of Fuzzy Sets Constituting a Content of Medical Linguistic Variables", In: Proceedings of 2007 IEEE Conference on Fuzzy Systems, Imperial College, London July 2007, IEEE Computational Intelligence Society, 2007, pp. 924-929.

[10] F. Kacher, and M. Larbani, "Existence of Equilibrium Solution for a Non-cooperative Game with Fuzzy Goals and Parameters”, Fuzzy Sets and Systems, vol.159, 2008, pp. 164176.

[11] E. Rakus-Andersson, M. Salomonsson, and $H$. Zettervall, "Two-person Games with Fuzzy Entries of the Payoff Matrix”, accepted for FLINS 2008. 